\title{
Cuidado centrado na família em neonatologia: percepções dos profissionais e familiares
}

\author{
Family-centered care in neonatology: health workers' and families' perceptions
}

Atención centrada en la familia en neonatologia: percepciones de los profesionales y familiares

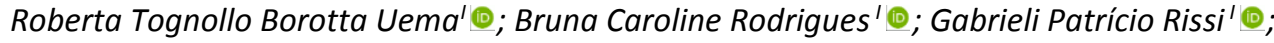 \\ Larissa Carolina Segantini Felipin' ‘; leda Harumi Higarashi' @ \\ 'Universidade Estadual de Maringá, Maringá, PR, Brasil
}

\begin{abstract}
RESUMO
Objetivo: analisar, sob a ótica dos profissionais que atuam em unidade de terapia intensiva neonatal e dos pais das crianças internadas, o entendimento do cuidado centrado na família. Método: estudo quantitativo de abordagem descritiva, realizado em 2018 com 19 profissionais de enfermagem de um hospital de ensino na região noroeste do Paraná e nove familiares. Utilizaram-se dois questionários autoaplicáveis acerca do tema e os sujeitos foram abordados durante seu período de permanência na unidade. Os dados foram analisados por estatística descritiva. Pesquisa aprovada pelo comitê de ética em pesquisa da instituição. Resultados: a equipe demonstra acolhimento pela família, os pais sentem esse vínculo, porém nos momentos de tomada de decisão, esta ainda é centralizada no profissional de saúde. Conclusão: a percepção dos profissionais e dos familiares converge para o cuidado centrado na família, porém, de forma incipiente, desconhecendo seus demais desdobramentos e maneiras de colocá-lo em prática.
\end{abstract}

Descritores: Cuidados de Enfermagem; Família; Unidades de Terapia Intensiva Neonatal; Relações Profissional-Família.

\section{ABSTRACT}

Objective: to examine family-centered care as seen by health personnel working in a neonatal intensive care unit and the parents of children hospitalized there. Method: this quantitative, descriptive study was conducted in 2018 with 19 nursing personnel at a teaching hospital in northwest Paraná State and nine family members. Two self-administered questionnaires on the topic were used, and study subjects were approached during their time at the unit. Data were analyzed using descriptive statistics. The study was approved by the institution's research ethics committee. Results: the team demonstrated acceptance by the family, and the parents felt that bond, although decision making still centered on the health professional. Conclusion: the health personnel's and patient relatives' perceptions converge to family-centered care, although in an incipient manner, and they are unaware of how it can be further developed and put into practice.

Descriptors: Nursing Care; Family; Intensive Care Units, Neonatal; Professional-Family Relations.

\section{RESUMEN}

Objetivo: analizar la atención centrada en la familia como la ve el personal de salud que trabaja en una unidad de cuidados intensivos neonatales y los padres de los niños hospitalizados allí. Método: este estudio cuantitativo y descriptivo se realizó en 2018 con 19 miembros del personal de enfermería de un hospital universitario del noroeste del estado de Paraná y nueve familiares. Se utilizaron dos cuestionarios autoadministrados sobre el tema y se abordó a los sujetos de estudio durante su tiempo en la unidad. Los datos fueron analizados utilizando estadística descriptiva. El estudio fue aprobado por el comité de ética en investigación de la institución. Resultados: el equipo demostró aceptación por parte de la familia y los padres sintieron ese vínculo, aunque la toma de decisiones aún se centró en el profesional de la salud. Conclusión: las percepciones del personal de salud y familiares del paciente convergen hacia la atención centrada en la familia, aunque de manera incipiente, y desconocen cómo se puede desarrollar y poner en práctica.

Descriptores: Atención de Enfermería; Familia; Unidades de Cuidados Intensivos Neonatal; Relaciones Profesional-Família.

\section{INTRODUÇÃO}

Atualmente vive-se, no âmbito da saúde, uma mudança de paradigma no contexto dos cuidados, onde o enfoque, anteriormente centrado no curativismo, hoje assume um papel muito mais amplo, trazendo para dentro do ambiente hospitalar o cuidado centrado no paciente e sua família, momento este em que à humanização do atendimento e a qualidade de vida tornam-se prioridade, diminuindo a atenção específica para o processo do adoecimento. Essa nova abordagem é traduzida como o Cuidado Centrado na Família (CCF), cuja característica engloba uma filosofia de cuidados prestados ao paciente e sua família, dando voz à ambos ${ }^{1}$.

Tal modelo utiliza pressupostos que devem ser seguidos durante toda a hospitalização seja ela no contexto neonatal, pediátrico ou adulto. Os principais incluem a aplicação: dignidade e respeito (os profissionais de saúde 
respeitam as escolhas, valores e crenças do paciente e da família); informação compartilhada (os profissionais comunicam e dividem as informações de maneira completa e imparcial com os pacientes e a família); participação (pacientes e famílias são encorajados e apoiados a participar da tomada de decisão); e colaboração (pacientes e famílias são incluídos e entendidos como base de apoio da instituição, auxiliando no desenvolvimento de políticas e programas, na educação profissional e na prestação do cuidado) $)^{2}$.

Especificamente no contexto neonatal, os benefícios do CCF, estão diretamente relacionados à redução do estresse parental e ao aumento da autoconfiança no trabalho, diminuição do tempo de internação do bebê e de reinternações, maior adesão ao método canguru, fortalecimento do vínculo entre recém-nascido e família e aumento nas taxas de aleitamento materno ${ }^{3,4}$.

Ao mesmo tempo, encontramos na ótica dos pais, pouca noção sobre a importância da sua real participação no cuidado do filho hospitalizado e que estes podem e devem ser empoderados sobre o estado de saúde e situação clínica dos seus filhos. $\mathrm{O}$ fato de as famílias poderem expressar seus sentimentos e medos, perante a internação do bebê em UTIN, faz com que boa parte dos pressupostos do CCF sejam aplicados, ainda que de forma indireta ${ }^{2}$.

Embora apresente diversas vantagens, o CCF ainda é pouco conhecido e aplicado no nosso país. Esse desconhecimento pelo tema influência de forma negativa na assistência ao bebê e sua família. Grande parte dos profissionais não só não sabe acerca do tema e seus pressupostos, como também, alega diversas barreiras para não o fazê-lo em seu ambiente de trabalho. Soma-se a isso, o fato de o CCF não ser discutido durante a formação acadêmica do enfermeiro e posteriormente em sua vida profissional ${ }^{5}$.

Alguns estudos reforçam a necessidade produzir evidências que sustentem a aplicação do CCF na prática, tornando necessária a utilização de instrumentos de medida que consigam apontar especificamente como os profissionais percebem a aplicação desse modelo em sua unidade ${ }^{6}$.

As ferramentas Shields \& Tanner Questionnaires ${ }^{7,8}$ são alguns dos instrumentos desenvolvidos para medir e comparar as perspectivas de pais e de profissionais de saúde sobre o CCF em diferentes contextos pediátricos. Trata-se de questionários estruturados e autoaplicáveis para pais e profissionais de saúde e as questões são correspondentes, de maneira que comparações podem ser feitas entre os dois grupos. Os dois questionários foram denominados Perceptions of Family Centered Care Parent (PFCC-P) e Perceptions of Family Centered Care-Staff (PFCC-S), traduzidos para o Brasil como: Percepção do Cuidado Centrado na Família-Equipe (PCCF-E) e Percepção do Cuidado Centrado na Família-Pais (PCCF-P)

Nesse contexto, surgem alguns questionamentos, tais como: qual o real entendimento da equipe de enfermagem sobre a participação da família no ambiente da Unidade de Terapia Intensiva Neonatal (UTIN) e de que forma estes aplicam ainda que de forma indireta o CCF? Ao mesmo tempo, como os pais entendem sua participação nesse processo e como poderiam melhorar seu vínculo e presença dentro da unidade e com outros membros da equipe?

Diante do exposto, o objetivo do estudo foi analisar, sob a ótica dos profissionais que atuam em unidade de terapia intensiva neonatal e dos pais das crianças internadas, o entendimento do cuidado centrado na família.

\section{MÉTOdo}

Trata-se de um estudo quantitativo de abordagem descritiva, realizado com sete enfermeiros e 12 técnicos de enfermagem que atuam em uma UTIN de um hospital de ensino situado na região noroeste do Paraná, Brasil. Ao mesmo tempo, realizou-se também aplicação do instrumento com nove familiares de bebês internados na unidade, sendo estes sete mães e dois pais.

Com relação ao local, trata-se de um Hospital Universitário situado no noroeste do estado do Paraná que atende exclusivamente o Sistema Único de Saúde. A UTIN já possui 20 anos de existência e tem como foco o tratamento de bebês de até 28 dias de vida, prematuros ou não. A unidade conta com 10 leitos neonatais, sendo quatro de cuidados intermediários e seis leitos de cuidados intensivos.

Utilizou-se como critérios de inclusão ter idade igual ou superior a 18 anos. Para os profissionais, também se exigiu experiência mínima de seis meses na área. Os critérios de exclusão abarcaram os participantes que estavam de férias ou de licença durante o período de coleta de dados, ou se recusaram a participar do estudo.

A equipe de enfermagem conta com 27 colaboradores, sendo 15 técnicas de enfermagem e 12 enfermeiras. Desse total, dois se recusaram a participar, três estavam de férias e três de licença, totalizando 19 participantes representando todos os turnos de trabalho. Os dados foram coletados entre os meses de fevereiro e maio de 2018, por meio da aplicação de questionários adaptados pelas pesquisadoras e baseados nos instrumentos: Percepção do Cuidado Centrado na Família-Equipe (PCCF-E) e Percepção do Cuidado Centrado na Família-Pais (PCCF-P) ${ }^{3}$. 
Tanto os profissionais como os familiares foram abordados durante seu período de permanência na unidade, em local reservado para tal atividade. Em um primeiro momento, o estudo era explanado ao profissional e ao pai ou mãe do bebê, posteriormente coletava-se anuência mediante assinatura do termo de consentimento livre e esclarecido (TCLE) e os sujeitos preenchiam o instrumento. Ao término da coleta, os dados foram analisados com auxílio de estatística descritiva, utilizando-se frequência relativa e absoluta.

Por se tratar de uma unidade relativamente pequena, com bebês que apresentam longo período de internação e pela limitação do tempo da coleta de dados, optou-se por criar dois instrumentos autoaplicáveis baseados no PCCF-P e PCCF-E, mas que pudesse ser analisado de uma forma descritiva e não utilizando uma escala likert, que é o proposto por Shields e Tanner 5 .

O projeto do estudo foi apreciado pelo Comitê Permanente de Ética em Pesquisa Envolvendo Seres Humanos, sob parecer no 2.092.136/2017 e Certificado de Apresentação para Apreciação Ética no 66242617.4.0000.0104. Todos os preceitos éticos da Resolução 466/2012 do Conselho Nacional de Saúde foram contempladas.

\section{RESULTADOS}

Dos 19 profissionais de enfermagem, todos eram do sexo feminino, sendo sete enfermeiras e 12 técnicas de enfermagem. A idade variou de 30 a 55 anos, com média de 42 anos. O tempo de atuação na unidade variou de dois até 23 anos, sendo que sete colaboradores atuavam na unidade há mais de vinte anos. Quanto a cursos de especialização, 11 já haviam realizado pós-graduação latu sensu na área da neonatologia e apenas uma possuía pósgraduação stricto sensu, em nível de mestrado em enfermagem.

Em relação aos familiares, dos nove sujeitos abordados, sete eram mães e dois eram pais. A idade variou entre 30 e 45 anos, com média de 37 anos. Somente dois pais possuíam curso superior completo e o restante enquadrava-se no ensino fundamental e médio completo. Cinco residiam no mesmo munício da unidade estudada e o restante morava nas cidades vizinhas com menos de $30 \mathrm{~km}$ de distância.

Metade dos familiares referiu certa dificuldade em conseguir chegar até a unidade para visitar seu bebê e a outra metade não sentia esse problema. Cinco deles gastavam cerca de 30 minutos para vir ao hospital e o restante até uma hora. A maioria, sete familiares, possuíam outras crianças para cuidar em casa, sejam elas filhos menores, ou crianças de outros relacionamentos do seu companheiro (a), cujas idades variaram entre seis e 12 anos. Esses mesmos sete referiram possuir ajuda em casa dos parentes (esposo, sogra, cunhada e enteada) para conseguir cuidar das outras crianças e ainda assim, conseguir visitar seu bebê na unidade.

Os resultados relacionados à equipe são descritos na Tabela 1.

TABELA 1: Rotinas do cuidado centrado na família realizadas pela equipe (n=19). Maringá, Paraná, Brasil, 2018.

\begin{tabular}{|c|c|c|c|c|}
\hline \multirow[t]{2}{*}{ Questões } & \multicolumn{2}{|c|}{ Sim } & \multicolumn{2}{|c|}{ Não } \\
\hline & $\mathbf{n}$ & $f(\%)$ & $\mathbf{n}$ & $f(\%)$ \\
\hline 1) Os pais são bem recepcionados quando chegam à unidade & 19 & $100 \%$ & 0 & 0 \\
\hline 3) Os pais podem acompanhar seu bebê durante os procedimentos. & 7 & $37 \%$ & 12 & $63 \%$ \\
\hline 4) Os pais tem livre arbítrio para questionar o tratamento do seu bebê. & 14 & $74 \%$ & 5 & $26 \%$ \\
\hline $\begin{array}{l}\text { 5) Os pais são preparados para a alta e sobre os encaminhamentos necessários após } \\
\text { a alta de seu bebê. }\end{array}$ & 15 & $79 \%$ & 4 & $21 \%$ \\
\hline 6) Os pais participam do processo de tomada de decisão sobre o cuidado. & 7 & $37 \%$ & 12 & $63 \%$ \\
\hline 7) O folder educativo entregue na admissão do bebê, é bem explicado aos familiares. & 12 & $63 \%$ & 7 & $32 \%$ \\
\hline 8) A família é incluída no cuidado do bebê. & 19 & $100 \%$ & 0 & 0 \\
\hline 9) A equipe conhece as necessidades de cada bebê. & 16 & $84 \%$ & 3 & $16 \%$ \\
\hline 10) A equipe possui tempo para ouvir e acolher as preocupações dos pais. & 16 & $84 \%$ & 3 & $16 \%$ \\
\hline
\end{tabular}

Os resultados encontrados demonstram que grande parte dos profissionais utiliza pressupostos do CCF em sua jornada de trabalho, visto que em 19 (100\%) casos os pais são bem acolhidos na unidade bem como são incluídos nos cuidados com o bebê. Ao mesmo tempo, nota-se que nem sempre outros familiares são bem recebidos no ambiente. $O$ instrumento aplicado não especificava qual vínculo esses outros familiares tinham com o bebê, porém isso não justifica o fato de 7 (32\%) profissionais não os receberem de forma adequada, conforme demonstrado na Tabela 1. 
Ainda, identificou-se que16 colaboradores (84\%) conhecem as necessidades de cada bebê e tem tempo para ouvir e acolher a preocupação dos pais. Sobre a tomada de decisão em relação ao cuidado, somente 7 profissionais (32\%) alegaram que os pais participam desse momento, porém quando questionados sobre o livre arbítrio da família para questionar o tratamento oferecido, 14 (74\%) informaram que esse é um direito dos pais.

Os resultados relacionados à percepção dos pais sobre a aplicação do instrumento, assim como aqueles relativos à equipe são descritos na Tabela 2.

TABELA 2: Percepção dos pais sobre cuidado centrado na família quando chegam à unidade ( $n=19)$. Maringá, Paraná, Brasil, 2018.

\begin{tabular}{|c|c|c|c|c|}
\hline \multirow[t]{2}{*}{ Questões } & \multicolumn{2}{|c|}{ Sim } & \multicolumn{2}{|c|}{ Não } \\
\hline & $\mathbf{n}$ & $\mathbf{f}(\%)$ & $\mathbf{n}$ & $f(\%)$ \\
\hline 1) Me sinto bem quando venho ao hospital. & 9 & $100 \%$ & 0 & 0 \\
\hline 2) Outras pessoas da minha família são bem recebidas na instituição. & 6 & $67 \%$ & 3 & $33 \%$ \\
\hline 3) Eu consigo acompanhar meu bebê durante os procedimentos. & 4 & $44 \%$ & 5 & $56 \%$ \\
\hline 4) Eu consigo fazer perguntas sobre o tratamento do meu bebê. & 7 & $78 \%$ & 2 & $22 \%$ \\
\hline $\begin{array}{l}\text { 5) Me sinto preparado para a alta e para cuidar do meu bebê em casa. Sei aonde encaminhá-lo } \\
\text { quando necessário. }\end{array}$ & 8 & $89 \%$ & 1 & $11 \%$ \\
\hline 6) A equipe me inclui nas decisões a serem tomadas sobre o meu bebê. & 7 & $78 \%$ & 2 & $22 \%$ \\
\hline 7) Eu compreendo as orientações escritas que recebi no folder admissional... & 7 & $78 \%$ & 2 & $22 \%$ \\
\hline 8) Eu recebo orientações sobre os cuidados do meu bebê.. & 8 & $89 \%$ & 1 & $11 \%$ \\
\hline 9) A equipe conhece as necessidades do meu bebê e dos outros que estão internados. & 8 & $89 \%$ & 1 & $11 \%$ \\
\hline 10) A equipe ouve minhas preocupações e entende o que eu e minha família estamos passando. & 9 & $100 \%$ & 0 & 0 \\
\hline
\end{tabular}

Todas as famílias abordadas alegaram que a UTIN estudada é um ambiente que os recebe bem e sabe ouvir suas necessidades e preocupações. Na maioria das situações, 8 (89\%) os pais referiram que são inclusos nos cuidados, bem como nas decisões tomadas acerca da situação de seu filho. Quando questionados sobre a participação durante os procedimentos, somente 4 (44\%) informaram que podiam acompanhar seu bebê.

\section{DISCUSSÃO}

Pode-se notar que a equipe se preocupa em recepcionar de forma agradável os familiares quando estes chegam à unidade e esta também é uma percepção da família. Alguns autores destacam a importância da enfermagem em recebê-los de forma acolhedora quando estes chegam até à unidade, para amenizar o impacto de estar em um local desconhecido e que por este motivo pode ser provedor de más impressões ${ }^{9}$.

Em relação à tomada de decisões e realização de procedimentos, grande parte da equipe, $63 \%$, refere que os pais não têm participação nesses momentos e esta foi uma realidade retratada pelos mesmos, reforçado o modelo curativista e centrado no profissional e não na família.

Garantir um bom entendimento do estado de saúde do filho, percebendo se os pais estão compreendendo os cuidados prestados e se esforçar para que estes esclareçam suas dúvidas ajuda na diminuição da ansiedade e auxilia na construção da confiança que nasce entre a equipe e a família. Quando o CCF é aplicado, é notável a diminuição dos níveis de estresse, ansiedade e depressão nos familiares, ao mesmo tempo em que a satisfação e o bom relacionamento com os profissionais de saúde aumentam. Para os profissionais, a prática pode melhorar sua satisfação e confiança no trabalho, a qualidade do atendimento e diminuir o esgotamento mental ${ }^{10}$.

A equipe refere que a família pode questionar o cuidado que foi realizado em seu filho (74\%), porém eles não participam da tomada de decisões, o que pode levar a crer, que a família é informada do procedimento ou conduta que foi realizada, pode perguntar sobre, mas este já aconteceu, portanto não interfere de forma direta na realização do mesmo. Ao mesmo tempo, os pais referem (78\%) que participam desse processo, supondo-se que esta não compreende seu papel enquanto responsável pelo bebê e que tem liberdade para interferir nesses momentos. $O$ fato de ser informada do que já aconteceu, não significa participar da tomada de decisão, portanto, supõe-se que faltam explicações adequadas à família, sobre seu papel nessas condutas.

O compartilhamento das informações e dos saberes profissionais sobre o quadro clínico do bebê, sua rotina de sono e repouso, evolução do padrão respiratório, aspectos nutricionais, eliminações e demais situações que acontecem dentro da unidade fazem parte dos anseios da família. Quando estes têm acesso a tais informações e sobre suas dúvidas, os pais passam a se tornar mais capazes de lidar com a situação da prematuridade e adoecimento, e começam a enfrentar melhor os momentos difíceis e de fragilidade ${ }^{9}$. 
A comunicação eficaz é a chave para facilitar o CCF. Os profissionais devem estar disponíveis para fornecer apoio durante os processos e intercorrências que permeiam a internação do bebê, bem como no acolhimento à família desde o primeiro momento ${ }^{11}$.

Em relação ao preparo para alta hospitalar e encaminhamento para acompanhamento ambulatorial do bebê, este também foi uma preocupação por parte da equipe e a família sentiu esse cuidado de uma forma positiva, entretanto não sabemos como de fato ele ocorre se começa no momento da internação do bebê, ou se é apenas algo focalizado no momento da saída do hospital.

A alta é um momento rodeado de expectativa e emoção, porém ao mesmo tempo em que se torna um dia feliz, este momento também pode ser motivo de apreensão, insegurança e medo. Dessa forma, torna-se primordial que a família esteja preparada para receber esse bebê em casa e tenha uma rede de apoio que consiga apoiá-la e encorajála. É importante que os pais exerçam o cuidado do seu filho ainda durante a internação para que no momento da ida para a casa, estes estejam seguros e sintam-se capazes de cuidar do seu bebê, sabendo aonde recorrer caso seja necessário ${ }^{12}$.

Sobre o folder explicativo entregue na admissão na unidade, percebe-se que existe um bom entendimento de ambos os lados. Os profissionais referem que o fazem de forma adequada e a família relata que consegue entender as explicações. Durante a coleta de dados, a pesquisadora não teve acesso direto ao folder, mas conversando com a coordenação da unidade, percebeu-se que se trata de um impresso com os horários de visita e funcionamento da UTIN, a importância dos pais dentro da unidade durante a hospitalização do bebê e informações sobre o Banco de Leite Humano do hospital caso seja necessário.

Trata-se de informações pontuais e que devem ser reforçadas durante o período de internação, pois na admissão a família geralmente está emocionalmente abalada, visto que a maioria das gestantes não almeja um parto prematuro, ocorrido por problemas maternos ou fetais e que culminaram em uma internação em UTIN. Em muitos casos, os avisos não são absorvidos e os familiares precisam de um momento de adaptação a essa nova realidade que não estava nos seus planos, mas que por algum motivo específico acabou acontecendo.

Uma estratégia para auxiliar na compreensão das informações repassadas é a entrega de um diário, intitulado Diário do Bebê, cuja finalidade não se baseia apenas em informar, mas sim tornar-se um registro das emoções maternas e seus sentimentos durante a internação. Além disso, também são abordados temas que permeiam a UTIN, como os equipamentos, principais patologias decorrentes da prematuridade, orientações sobre aleitamento materno, vacinas e preparo para alta, e fornece um espaço para anotações sobre ordenha do leite, carimbo da mão e pé do bebê, dados do nascimento e ser um espaço de apoio com registro do cotidiano vivenciado para auxiliar no enfrentamento da situação ${ }^{13}$.

Isso reforça a importância da equipe ser humanizada e trabalhar os pressupostos do CCF durante o processo, lembrando que a família não pediu para estar naquela situação, o bebê não deveria ter nascido antes da hora, somado a outros fatores que permeiam esse momento, como a distância do hospital, o fato de ter outros filhos pequenos em casa, dificuldades financeiras que interferem na visitação do recém-nascido, e até mesmo, um luto pelo filho que não estava esperado naquele momento.

Estudos que foquem em investigar a satisfação dos pais durante o período de internação, são determinantes para aprimorar as práticas do cuidado e consequentemente auxiliam na implantação dos pressupostos do CCF $^{14}$.

Nesse contexto, a enfermagem exerce suma importância, pois acolher a família diante das suas necessidades auxiliando a diminuir o estresse a traçar estratégias para facilitar o enfrentamento da hospitalização do seu filho faz com que aconteça uma diminuição da ansiedade e facilite a formação do vínculo ${ }^{10}$.

Às vezes os pais estavam esperando que o bebê nascesse só dali dois ou três meses, e de repente se veem numa situação como essa. É preciso muita sensibilidade e empatia por parte da equipe para enxergar a família com esses olhos, lembrando que esta não está ali para piorar a situação ou para torna-la mais difícil do que já é, mas sim para acolher, ouvir, e fazer com que esse momento seja o menos sofrido possível. Esses aspectos foram reforçados pelos familiares, pois tanto a equipe (84\%) como a família (100\%) refere esse acolhimento e escuta.

A gestação é um período de grande expectativa, por parte da família e os pais anseiam por um parto tranquilo e para que o nascimento do filho ocorra da forma mais saudável possível. Entretanto, a internação em UTIN traz consigo sentimentos de insegurança e desamparo, reforçando a necessidade de acolher a família nesse momento ${ }^{15}$.

O acolhimento deve ser uma forma de operacionalizar o trabalho em saúde, mantendo uma postura de escuta, comprometida a responder os questionamentos e a auxiliar na resolução das necessidades expressadas pela família, assumindo a responsabilidade de garantir uma assistência adequada e centrada na família ${ }^{16}$. 
Sobre a participação nos cuidados com o bebê, ambos referem que isso acontece, o que se torna algo positivo durante a hospitalização, pois em alguns casos a mãe principalmente, pode sentir que ainda não é a responsável por aquele bebê, uma vez que ele nasceu, foi para unidade cheia de fios e tubos e ela não consegue acarinhá-lo ou alimentálo por exemplo. O fato de ordenhar o leite materno a beira leito e ajudar a administrá-lo via sonda orogástrica, tocar seu bebê, ou simplesmente estar ali por ele, faz com que aos poucos a família inicie esse processo de apropriação do cuidado e aos poucos, esta torna-se mais fortalecida e segura. Cabe à equipe perceber essas dificuldades de aproximação e estimular para que o vínculo aconteça da melhor forma possível, pois beneficiará ambas as partes.

A participação familiar precoce com incentivo ao toque e a pequenas ações como auxílio na troca de fraldas predispõe a um bom processo de enfrentamento da condição do filho e ajuda a fornecer segurança no momento de levar o bebê para casa ${ }^{17}$. As intervenções centradas no CCF podem não ter impacto significativo na mortalidade, porém pode ajudar a diminuir o tempo de internação na UTIN ${ }^{18}$.

Nota-se que não houve discrepância entre as respostas positivas da equipe e da família, fato que reforça a caracterização da unidade como humanizada e engajada no processo de cuidar do bebê e sua família. Sobre a tomada de decisão e participação da família nos procedimentos, percebe-se que este ainda é um caminho a ser percorrido, pois a família nem sempre está psicologicamente preparada para vivenciar esse momento e a equipe pode não saber como lidar com eles nesse processo. Sugere-se que isso comece aos poucos e de uma forma que seja confortável para ambos.

Um estudo que trata da participação familiar em momentos críticos traz que a família enxerga isso como algo positivo, pois conseguiu ver de forma clara como funciona o atendimento ao seu ente querido e mesmo quando o desfecho foi o óbito, esse momento tornou-se menos angustiante, trazendo de certa forma, uma sensação de conforto no momento de dor ${ }^{5}$.

Entende-se que isso não pode ser generalizado e que nem todas as instituições têm perfil e profissionais qualificados para acolher a família nesse momento, ao mesmo tempo nem todos os pais estão preparados para participar disso. Porém, sugere-se que a discussão seja válida, e que de alguma forma deva ser iniciada, dentro da própria unidade neonatal.

\section{Limitações do estudo}

O número de participantes no estudo torna-se uma limitação, e a própria característica da unidade corrobora para que o n não seja alto. Sugere-se a realização de outros estudos com tempo maior de acompanhamento da família e da equipe, para tentar visualizar de forma mais prática os pressupostos do CCF.

\section{CONCLUSÃO}

Considerando os dados desse estudo, conclui-se que os profissionais têm pouco entendimento sobre o real significado do CCF. Para eles, sua prática envolve alguns dos pressupostos do CCF, entretanto, este é realizado ainda que de forma indireta e não completa. Em relação à família, a mesma não tinha conhecimento sobre o que seria o CCF, porém se sente acolhida e participativa no cuidado, mesmo que grande parte das decisões seja tomada pelos profissionais sendo somente comunicadas posteriormente a ela.

Ressalta-se a necessidade de incluir a família nesse processo, dar voz àqueles que são os responsáveis legais da criança e que em sua grande maioria não conhecem seus direitos e sua importância nesse momento. Ao mesmo tempo, sugere-se a necessidade de incluir o CCF na prática profissional da equipe, a fim de diminuir a lacuna entre o que é preconizado e o que realmente acontece na assistência.

\section{REFERÊNCIAS}

1. Balbino FS, Balieiro MMFG, Mandetta MA. Measurement of Family-centered care perception and parental stress in a neonatal unit. Rev. latinoam. enferm. (Online). 2016 [cited 2019 Sept 05]; 24:e2753. DOI: https://doi.org/10.1590/1518-8345.0710.2753.

2. Felipin LCS, Merino MFGL, Baena JA, Oliveira RBSR, Borghesan NBA, Higarashi IH. Family-centered care in neonatal and pediatric intensive care unit: nurse's vision. Ciênc. cuid. saúde. 2018 [cited 2019 Sept 05]; 17(2):1-7. DOI: https://doi.org/10.4025/cienccuidsaude.v17i2.41001.

3. Sampaio AA, Zonta JB, Ferreira FY, Okido ACC. Family-centered care in a pediatric intensive care unit: professionals' perceptions. Rev. Rene (Online). 2017 [cited 2019 Sept 04]; 18(4):515-20. DOI: https://doi.org/10.15253/21756783.2017000400013

4. Sousa FCP, Montenegro LC, Goveia VR, Corrêa AR, Rocha PK, Manzo BF. Family participation in patient safety in neonatal units from the nursing perspective. Texto \& contexto enferm. 2017 [cited 2019 Sept 06]; 26(3):e1180016. DOI: https://doi.org/10.1590/0104-07072017001180016.

5. Barreto MS, Arruda GO, Garcia-Vivar C, Marcon SS. Family centered care in emergency departments: perception of brazilian nurses and doctors. Esc. Anna Nery Rev. Enferm. 2017 [cited 2019 Sept 07]; 21(2):e20170042. Available from: https://www.scielo.br/scielo.php?pid=S1414-81452017000200213\&script=sci_arttext\&tlng=en. 
6. Silva T, Alves LB, Balieiro MM, Mandetta MA, Tanner A, Shields L. Cross-cultural adaptation of an instrument to measure the family-centered care. Acta paul. enferm. 2015 [cited 2019 Sept 07]; 28(2):107-12. DOI: https://doi.org/10.1590/19820194201500019.

7. Shields L. Questioning family - centered care. J. clin. nurs. 2010 [cited 2019 Sept 12]; 19(17):2629-38. DOI: https://doi.org/10.1111/j.1365-2702.2010.03214.x.

8. Shields L, Tanner A. Pilot study of a tool to investigate perceptions of family-centered care in different care settings. J. pediatr. nurs. 2004 [cited 2019 Sept 12]; 30(3):198-9. Available from: https://www.ncbi.nlm.nih.gov/pubmed/15311642.

9. Soares LG, Soares LG, Decesaro MN, Higarashi IH. Perception of families on reception in the neonatal context during an intervention process. Rev. pesqui. cuid. fundam. (Online). 2019 [cited 2019 Sept 12]; 11(1):147-53. Available from: http://www.seer.unirio.br/index.php/cuidadofundamental/article/view/6577.

10. Park M, Giap TT, Lee G, Jeong H, Jeong M, Go Y. Patient- and family-centered care interventions for improving the quality of health care: A review of systematic reviews. Int. J. Nurs. 2018 [cited 2020 Oct 04]; 87: 69-83. DOI: https://doi.org/10.1016/j.ijnurstu.2018.07.006.

11. Foster M, Whitehead L, Maybee P. The Parents', hospitalized child's, and health care providers' perceptions and experiences of family-centered care within a pediatric critical care setting: a synthesis of quantitative research. J. Fam. Nurs. 2016 [cited 2020 Oct 04]; 22(1). DOI: https://doi.org/10.1177/1074840715618193.

12. Silva RMM, Menezes CCS, Cardoso LL, França AFO. Experiences of families neonate early in hospitalized neonatal intensive care unit: integrative review. Rev. enferm. Cent.-Oeste Min. 2016 [cited 2019 Sept 12]; 6(2):2258-70. DOI: https://doi.org/10.19175/recom.v6i2.940.

13. Leite CCP, Souza SNDH, Rossetto EG, Pegoraro LGO, Jacinto VCB. The Baby's Diary to the premature infant's mother: supporting family-centered care. Rev. enferm. UERJ. 2016 [cited 2019 Sept 13]; 24(1):e8664. DOI: https://doi.org/10.12957/reuerj.2016.8664.

14. Regan KM, Curtin C, Vorderer L. Paradigm shifts in inpatient psychiatric care of children: Approaching child- and family-centered care. J. Child Adolesc. Psychiatr. Nurs. 2018 [cited 2020 Oct 04]; 30(4): 186-194. DOI: https://doi.org/10.1111/jcap.12193.

15. Kegler JJ, Neves ETA, Silva AM, Jantsch LB, Bertoldo CS, Silva JH. Stress in parents of newborns in a Neonatal Intensive Care Unit. Esc. Anna Nery Rev. Enferm. 2019 [cited 2019 Sept 12]; 23(1):e20180178. DOI: https://doi.org/10.1590/2177-9465-ean-20180178.

16. Al Maghaireh DF, Abdullah KL, Chan CM, Piaw CY, Al Kawafha MM. Systematic review of qualitative studies exploring parental experiences in the Neonatal Intensive Care Unit. J. clin. nurs. 2016 [cited 2019 Sept 12]; 25(19-20):2745-56. DOI: https://doi.org/10.1111/jocn.13259.

17. Schmidt KT, Sassá AH, Veronez M, Higarashi IH, Marcon SS. The first visit to a child in the neonatal intensive care unit: parents perception. Esc. Anna Nery Rev. Enferm. 2012 [cited 2019 Sept 12]; 16(1):73-81. DOI: https://doi.org/10.1590/S141481452012000100010.

18. Goldfarb MJ, Bibas L, Bartlett V, Jones H, Khan N. Outcomes of patient- and family-centered care interventions in the ICU: a systematic review and meta-analysis. Crit. Care Med. 2017 [cited 2020 Oct 04]; 45(10): 1751-1761. DOI: https://doi.org/10.1097/CCM.0000000000002624. 